\title{
Liderança na educação médica
}

\author{
Leadership in medical education
}

\author{
Liderazgo en la educación médica
}

\author{
Bruna C. Provenzano, Diogo A. V. Ferreira, André P. G. Machado, ${ }^{*}$ Renata N. Aranha
}

\begin{abstract}
Resumo
O modelo de saúde contemporâneo implicou modificações na medicina. Exige-se do médico uma formação generalista e ultrapassa-se a exigência técnica, incorporando-se novos atributos, dentre os quais se destaca a liderança. Desde 2001, as Diretrizes Curriculares do Curso de Medicina, do Conselho Nacional de Educação, definiram as competências que devem ser desenvolvidas durante a graduação, tornando a escola médica responsável por estimular, entre os alunos, a comunicação, a tomada de decisões e o trabalho em equipe, elementos essenciais à liderança. Embora mais de uma década tenha se passado, permanece uma notável lacuna no seu desenvolvimento. De fato, o seu caráter subjetivo dificulta a sua inserção no currículo formal. Todavia, ela é considerada, atualmente, um conceito multifacetado, que traspassa a ideia de qualidade inata à personalidade, sendo enxergada como uma competência que pode ser ensinada. Portanto, surge um desafio às escolas médicas: como ensinar liderança aos seus alunos? Nesse contexto, as atividades extracurriculares apresentam-se como estratégia de aprendizado que coloca o estudante frente a situações de gestão e trabalho em equipe. Dentre essas atividades, destacam-se as organizações estudantis, como as ligas acadêmicas, em que grupos de alunos, sob orientação docente, planejam e desenvolvem diversas atividades. Paralelamente, as empresas juniores são um exemplo similar às ligas, porém mais consolidadas e, por isso, vistas como fonte de experiência para estas. Logo, é fundamental o reconhecimento da legitimidade desses projetos e a abertura de espaço livre no currículo formal que possibilite cada vez mais a participação dos alunos em sua trajetória à formação profissional mais completa.
\end{abstract}

Descritores: Liderança; Educação médica; Organizações estudantis.

\begin{abstract}
The contemporary health model has brought changes to medical education. It demands a generalist training from doctors and transcends the technical needs, incorporating new attributes, among which leadership gains prominence. Since 2001, following these changes, the curricular guidelines of the medicine course set by Brazil's National Education Council established the competences to be developed throughout graduation, with the medical school being responsible for stimulating communication, decision making, and teamwork, essentials for leadership. Although it's been a decade, a lacuna in the development of this competence persists. In fact, its subjective character makes its inclusion in the formal curriculum difficult. However, it is considered today as a multifaceted concept beyond the idea of an innate personality quality, seen as a competency that can be taught. Thus, a new challenge arises for the medical school: how can leadership be taught? In this context, extracurricular activities present themselves as a learning strategy that put the students in management and teamwork situations. Among those, student organizations such as academic leagues, in which groups of students plan and develop a wide range of activities under faculty supervision, stand out. In parallel, junior enterprises present a similar example, albeit a more consolidated one, and are for this very reason seen as an experience source for the academic leagues. Therefore, essential are the recognition of the legitimacy of these projects and the inclusion of free time in the formal curriculum in order to allow the students' participation in a broader professional training.
\end{abstract}

Keywords: Leadership; Medical education; Student organizations.

Revista HUPE, Rio de Janeiro, 2014;13(4):26-31 Recebido: 23/05/2014 | Aprovado: 23/09/2014 doi: 10.12957/rhupe.2014.13943
Endereço para correspondência: Departamento de Clínica Médica, HUPE, UERJ. Boulevard 28 de Setembro, 77, sala 329, Rio de Janeiro, RJ, Brasil. CEP: 20551-030. E-mail: bcpro13@yahoo.com.br 


\section{Resumen}

El modelo de salud contemporáneo implica modificaciones en la formación médica. Se requiere médicos con una formación generalista. Por otra parte, se va mas allá de la exigencia técnica, incorporándose nuevos atributos, entre los que se destaca el liderazgo. Desde 2001, acompañando esas modificaciones, las Directrices Curriculares del Curso de Medicina, del Consejo Nacional de Educación, definieron las competencias y habilidades que se deben desarrollar durante la graduación, transformando a la escuela de medicina responsable de la estimulación, entre los estudiantes, la comunicación, la toma de decisiones y el trabajo en equipo, elementos esenciales en el liderazgo. Aunque haya pasado más de una década, sigue existiendo una brecha en su desarrollo. En cierto modo, su carácter subjetivo dificulta su inserción en el plan de estudios formal. Al mismo tiempo, se considera actualmente un concepto multifacético, que va mas allá de la idea de cualidad innata a la personalidad individual, siendo percibida como una competencia que se puede enseñar. Por lo tanto, surge un nuevo desafío para las escuelas de medicina: cómo enseñar liderazgo a sus estudiantes? En ese contexto, las actividades extracurriculares se presentan como estrategia de aprendizaje práctico que sitúa al estudiante en situaciones de gestión y trabajo en equipo. En el ámbito de estas actividades, se destacan las organizaciones estudiantiles como, por ejemplo, las ligas académicas en el área de salud, donde grupos de estudiantes, bajo la orientación de un profesor, planifican y desarrollan una amplia gama de actividades. En otros campos, las empresas júnior son un ejemplo similar a las ligas, pero más consolidadas y, por eso consideradas como fuente de experiencia para éstas. Por lo tanto, es fundamental reconocer la legitimidad de esos proyectos y abrir espacio libre en el plan de estudios formal que posibilite y estimule cada vez más la participación de los alumnos en su trayectoria para una formación profesional más completa.

Palabras clave: Liderazgo; Educación médica; Ligas académicas.

\section{Liderança na educação médica}

A educação médica vive uma mudança de paradigma curricular: há um importante movimento de reforma, cujo foco é a formação de um médico generalista com visão integral do paciente e voltada para atenção primária; entretanto, ainda há o tradicionalismo do modelo hospitalocêntrico, cujo alicerce é o aprendizado técnico-científico, valorizando a especialização e enfatizando a doença em contraposição ao doente. Sob o ponto de vista do Ministério da Educação, o novo currículo das escolas médicas deve acompanhar as mudanças sociais dos últimos anos, reforçadas pela implantação do Sistema Único de Saúde e pela reorganização da atenção primária centrada na Estratégia da Saúde da Família. O médico contemporâneo deve ter visão crítica, reflexiva e humanista, aliando o saber técnico à integralidade do cuidado. Nesse contexto, exigem-se do médico competências e habilidades antes não tão valorizadas para que possa corresponder aos papéis e às responsabilidades desse novo modelo de saúde. ${ }^{1}$ A liderança e o trabalho em equipe são exemplos desta nova exigência. Acompanhando essas mudanças, as escolas médicas começaram a levá-las em consideração em suas reformas curriculares e assumiram o desafio de formar mé- dicos que não só compreendem o cuidado através do ser, como também se apresentam aptos a se tornarem novos líderes em saúde. ${ }^{2}$

O papel da liderança na medicina acompanha cada área de atuação do médico. O contexto de aplicabilidade desta competência é amplo, não só contemplando circunstâncias diárias do atendimento ambulatorial, da gestão hospitalar e da saúde pública, ${ }^{3}$ mas também incluindo momentos de risco de vida como o atendimento a uma parada cardiorrespiratória. ${ }^{4}$ Dessa forma, devido ao papel central que o médico exerce pela natureza de suas atividades profissionais, é imprescindível o desenvolvimento de liderança.

Nos Estados Unidos, em uma análise recente dos currículos de 131 faculdades de medicina, apenas $10 \%$ delas colocam a liderança como um objetivo a ser alcançado. ${ }^{5} \mathrm{Em} 2000$, foi elaborado o projeto Undergraduate Medical Education for The 21st Century (UME-21), cujo objetivo era incentivar novos modelos curriculares de graduação médica que tornassem o aluno apto a prover um cuidado acessível, de alta qualidade e a responder às novas demandas sociais. Foram estabelecidas nove áreas de conhecimento, dentre as quais se inclui a liderança. Dessa forma, a escola médica assume a responsabilidade pelo desenvolvimento desta competência. Contudo, somente oito 
escolas participantes incluíram estratégias para contemplá-la em seu novo currículo médico, reiterando a lacuna existente no que diz respeito ao seu ensino. ${ }^{2}$

No Brasil, seguindo a tendência mundial, as Diretrizes Curriculares do Curso de Medicina (DCCM), implementadas desde 2001, definem o que é necessário para que o estudante atue de forma a unir a integralidade do atendimento com a tecnologia. Neste documento, são determinadas as competências e habilidades que esse novo médico deve desenvolver ao longo de sua formação. Ao todo, são seis competências gerais: 1) atenção em saúde; 2) tomada de decisões; 3) comunicação; 4) liderança; 5) administração e gerenciamento; 6) educação continuada. ${ }^{6}$

Dessa forma, surge a preocupação com o perfil do egresso da graduação. A faculdade deve, então, ultrapassar as barreiras do ensino médicobiológico centrado no modelo hospitalocêntrico, estimulando outras áreas tão importantes quanto a técnica. ${ }^{6}$ Entretanto, não diferindo do que ocorre no exterior, ainda há uma grande lacuna em relação à aprendizagem da liderança, mesmo nos mais novos currículos brasileiros. Surge, portanto, um novo desafio às escolas médicas: liderança e trabalho em equipe. Contudo, como desenvolver estas habilidades?

\section{Liderança: uma competência a ser ensinada}

O conceito de liderança é controverso, apesar de vastamente discutido por diversos especialistas, sendo essa uma das maiores dificuldades ao estudá-la. No campo da medicina, multifatorial e multifacetada, segundo as DCCM, "Liderança: no trabalho em equipe multiprofissional, os profissionais de saúde deverão estar aptos a assumirem posições de liderança, sempre tendo em vista o bem-estar da comunidade. A liderança envolve compromisso, responsabilidade, empatia, habilidade para tomada de decisões, comunicação e gerenciamento de forma efetiva e eficaz." ${ }^{6}$

Ao contrário do que se acredita, a liderança não é uma qualidade inata. Sendo uma competência, pode ser ensinada, envolvendo fatores cognitivos que podem e devem ser estimulados durante a graduação. ${ }^{7}$
A liderança engloba desde planejamento estratégico até comunicação, passando pelo trabalho em equipe e pela tomada de decisões. Os líderes devem ser capazes de desenvolver uma visão e traçar um objetivo e, em continuidade, transmitir esta visão à sua equipe, cativando-a de modo a levá-la a cooperar em prol deste objetivo. Dentro de um ambiente de saúde, boa liderança se reflete de forma positiva para os pacientes. Afinal, organiza-se um plano de ação e toda uma equipe é articulada para alcançar seus alvos: a saúde e o bem-estar da população. ${ }^{7,8}$

\section{Estratégias de aprendizado de liderança: o papel das atividades extracurriculares}

Diante desse cenário, algumas universidades americanas implementaram estratégias para o desenvolvimento da liderança. Uma das principais abordagens foi feita dentro das enfermarias durante as próprias rondas cotidianas, nas quais se discutia tanto questões clínicas, como também a dinâmica da equipe, o papel de cada profissional e o impacto que as ações coordenadas da equipe possuem no cuidado dos pacientes. ${ }^{2}$ De forma geral, os alunos mostraram boa aceitação em relação a estas atividades.

Existem poucas publicações sobre o ensino de liderança e a efetividade dessas práticas na formação médica. Contudo, é consenso que esta competência deve ser adquirida em um contexto prático, em que se desenvolva um método de aprendizagem no qual os alunos adotem postura ativa frente à própria formação. ${ }^{9}$ Como estratégia de aprendizado, as atividades extracurriculares, principalmente aquelas desenvolvidas e organizadas pelo corpo discente, assumem papel relevante no desenvolvimento de liderança durante a graduação. ${ }^{5}$

As atividades extracurriculares, passando ao largo dos processos de reforma e aprimoramento curricular, representam um importante espaço de ensino. Muitas vezes tidas apenas como um currículo paralelo, elas de forma alguma se limitam a isso: representam parte importante da formação dos futuros profissionais médicos, mesmo não sendo obrigatórias. Na verdade, é essa não obrigatoriedade que se traduz numa 
das características que fazem dessas atividades importante ferramenta de ensino: a busca ativa, voluntária, do conhecimento, com a construção de trajetórias mais autênticas e personalizadas para os alunos envolvidos. Desta forma, eles se sentem fazendo parte de sua própria formação profissional. A experiência permite aprendizado prático, possibilitando o senso de responsabilidade diante de uma equipe, o manejo de conflitos e até mesmo uma administração financeira. Além disso, há o aprendizado por pares, no qual o aluno aprende com outro aluno e, por sua vez, transmite o conhecimento adquirido a outros mais. ${ }^{5}$

\section{Liga acadêmica e empresa júnior como atividades extracurriculares}

Dois exemplos de movimentos conduzidos por jovens, em meio a um ambiente universitário muitas vezes marcado pela rigidez e estruturação formal das grades curriculares, merecem destaque: as ligas acadêmicas (LA) e as empresas juniores (EJ). Embora atuem sobre temas distintos, possuem em comum o caráter inovador e permitem a exploração de pontos não contemplados pelo currículo tradicional, como a liderança. ${ }^{10}$

Em relação às LA de medicina, embora não exista uma definição clara, algumas considerações podem ser levantadas. Nos dicionários, a palavra "liga", além de sinônimo de ligação, significa aliança, pacto." Uma LA é, antes de tudo, uma associação estudantil na qual alunos e professores pactuam em prol do fortalecimento da educação médica frente à sociedade do século $X X I$, abordando temas que muitas vezes não são contemplados nos currículos das universidades brasileiras. Os estudantes são os responsáveis pela gestão das ligas, sob a orientação de um ou mais professores. Desta forma, além de definirem uma vasta programação para o estudo da temática envolvida, são ainda inseridos na comunidade por meio de atividades educativas, preventivas ou de promoção da saúde, as quais são criadas e desenvolvidas por eles próprios. ${ }^{12}$

No ensino, as LA visam à capacitação dos estudantes para a atuação com a comunidade, para a aquisição de novos conhecimentos e para a produção científica. Objetivam o desenvolvimento do "saber fazer" e acontecem através de grupos de estudo, palestras, discussões de casos, cursos, simpósios e atividades de pesquisa e assistência em diferentes cenários da prática médica. Deve-se ressaltar, no entanto, que as LA não têm por objetivo a especialização precoce, mas sim complementar uma formação que a estrutura curricular não é capaz de promover, tendo em mente o conceito atual de saúde como bem-estar biopsicossocial e a formação generalista, conforme preconizado pelas DCCM. ${ }^{13}$

$\mathrm{Na}$ metodologia de trabalho das LA merece destaque a realização de atividades de extensão, que correspondem ao contato estudantescomunidade, transformando o aluno em um agente de promoção de saúde e transformação social. Assim, a liga representa um espaço de vivências que superam o simples assistencialismo, sendo, na verdade, uma troca de aprendizado entre os futuros profissionais médicos e a comunidade, objetivando a busca por projetos que atendam às demandas da população. Portanto, as ligas também representam um elemento para a construção da cidadania, formando indivíduos mais críticos e com visão social mais humanista. As LA também permitem a criação de um campo de diálogo entre realidades diversas dentro da sociedade brasileira tão repleta de contrastes, contribuindo para a aproximação entre a universidade e a comunidade. ${ }^{14}$

A empresa júnior (EJ) é uma organização discente, associada a um ou mais cursos da graduação ou do ensino técnico, encontrada, por exemplo, em escolas de engenharia e administração, onde já são consagradas e valorizam bastante o currículo pessoal do estudante participante. Sua gestão é realizada por alunos e supervisionada por um professor, os quais se organizam para montar uma empresa que atenda às demandas da sua comunidade, gerando um produto final. Apesar de levar em seu nome o conceito de empresa, seu objetivo final não é o lucro. De fato, a EJ oferece um espaço único durante a faculdade, pois permite ao aluno um aprendizado sobre como gerir projetos, como manejar orçamentos reduzidos, ou como lidar com uma equipe heterogênea, inserindo o estudante em situações reais. Um dos mais importantes aspectos é a questão de introduzir a prática durante o período da graduação, o qual muitas vezes se reserva apenas à teoria. Os alunos que participam acabam por adquirir habilidades e 
competências essenciais ao mercado de trabalho, como proatividade, criatividade, profissionalismo e compostura. As EJ ensinam aos alunos como liderar um projeto e como é fundamental a união de todos em prol do sucesso da empresa. ${ }^{10}$

Em certos campos fora da área da saúde, a liderança é um tema já inserido na formação acadêmica. Nesse ínterim, a EJ se constitui em uma das estratégias de desenvolvimento de liderança já consagradas, havendo inclusive o reconhecimento por parte do mercado de trabalho de que o aluno que participa desta modalidade de projeto tem um diferencial frente a outros que não participaram, abrindo-lhe oportunidades. Traçando-se um paralelo entre a EJ e a LA, esta seria uma proposta para a escola médica como aquela é para as de engenharia e administração. Como já dito, as EJ são bem estabelecidas. ${ }^{5}$ Em contraposição, as LA ainda buscam seu pleno reconhecimento dentro das faculdades de medicina, especialmente dentro da classe docente, na qual podemos verificar bastante desinformação a seu respeito, assim como resistência aos seus propósitos e às suas atividades. Desta forma, podemos dizer que as LA devem ver nas EJ características e métodos de desenvolvimento cuja maior experiência possa contribuir para seu futuro aprimoramento.

\section{Conclusão}

Novos paradigmas surgem dentro da formação acadêmica. Na medicina, há a preocupação de se formar um aluno competente não só na técnica, mas também em outras habilidades. Essas mudanças demandam modificações no currículo, o qual passa a contemplar novas áreas de conhecimento, como a liderança. Apesar de sua reconhecida importância, permanece uma marcante lacuna em relação à sua abordagem dentro do currículo médico brasileiro.

Por ser a liderança um requisito dentro das DCCM, as escolas médicas brasileiras devem assumir o compromisso de trabalhá-la durante a formação profissional. Entretanto, observa-se que a maior parte dos currículos em vigência não oferece ferramentas para isto.

Portanto, torna-se fundamental que a reforma curricular em andamento na Faculdade de Ciências Médicas considere, como parte de seu planejamento, a criação de espaços livres em sua grade horária que permitam ao aluno participar de forma plena desses grupos, reconhecendo as organizações estudantis como uma estratégia relevante de aprendizado. Não há dúvida de que, dessa forma, serão formados médicos melhor preparados para assumirem seus papéis de líderes na saúde brasileira.

\section{Referências}

1. Kaptzki D, Valente D, Matos JA, Aranha R. Introdução. Em: Proposta para uma graduação médica contemporânea. $1^{a}$ edição. KOAN. 2012. p.19-26.

2. O'Connell, Mark T, Pascoe, John M. Undergraduate medical education for the 21st century: leadership and teamwork. Fam Med. 2004;36 Suppl:S51-6.

3. Rabarison K, Ingram RC, Holsinger JW Jr. Application of Situational Leadership to the National Voluntary Public Health Accreditation Process. Front Public Health. 2013 Aug 12;1:26. http://dx.doi.org/10.3389/ fpubh.2013.00026

4. Hunziker S, Tschan F, Semmer NK, Marsch S. Importance of leadership in cardiac arrest situations: from simulation to real life and back. Swiss Med Wkly. 2013;143:w13774. http://dx.doi.org/10.4414/ SMW.2013.13774

5. Veronesi MC, Gunderman RB. Perspective: The Potential of Student Organizations for Developing Leadership: One School's Experience. Acad Med. 2012;87(2):226-9. http://dx.doi.org/10.1097/ ACM.0b013e31823fa47c

6. Conselho Nacional de Educação, Câmara de Educação Superior. Diretrizes Curriculares Nacionais do Curso de Medicina. Resolução Cne/Ces $N^{0} 4$, de 7 de Novembro de 2001.

7. Blumenthal DM, Bernard K, Bohnen J, Bohmer R. Addressing the leadership gap in medicine: residents' need for systematic leadership development training. Acad Med. 2012;87(4):513-22. http://dx.doi. org/10.1097/ACM.0b013e31824a0c47

8. Chakraborti C, Boonyasai RT, Wright SM, Kern DE. A systematic review of teamwork training interventions in medical student and resident education. J Gen Intern Med. 2008;23(6):846-53. http://dx.doi. org/10.1007/s11606-008-0600-6

9. Kiesewetter J, Schimdt-Huber M, Netzel J, Krohn AC, Angstwurm M, Fischer MR. Evaluiertes Training von Führungskompetenzen in der medizinischen Aus- und Weiterbildung. GMS Z Med Ausbild. 2013;30(4):Doc49. http://dx.doi.org/10.3205/ zma000892

10. Confederação Nacional de Empresas Junior, Conceito Nacional de Empresa Junior. Brasiljunior.org, 16 de junho 2012. 
11. Ferreira, ABH. Mini-Aurélio do Século XXI: o minidicionário da língua portuguesa. $4^{\mathrm{a}}$ ed. Rio de Janeiro: Nova Fronteira; 2000.

12. Azevedo RP, Dini PS. Guia para construção de Ligas Acadêmicas. Ribeirão Preto: Assessoria Científica da Direção Executiva Nacional dos Estudantes de Medicina, 2006. Disponível em: <http://www.daab.org. br/texto.asp?registro=157>. Acesso em: 15/05/2010.

13. Rodríguez CA, Kolling MG, Mesquita P. Educação em Saúde: um Binômio que Merece ser Resgatado. Rev Bras Educ Med. 2007;31(1):60-6

14. Ferreira DAV, Aranha RN, Souza MHFO. Ligas Acadêmicas: uma proposta discente para ensino, pesquisa e extensão. Interagir. 2011;16:47-51.

\section{Bruna C. Provenzano}

Faculdade de Ciências Médicas. Universidade do Estado do Rio de Janeiro. Rio de Janeiro, RJ, Brasil.

\section{Diogo A. V. Ferreira}

Programa de Pós-graduação em Ciências Médicas.

Faculdade de Ciências Médicas. Universidade do Estado do Rio de Janeiro. Rio de Janeiro, RJ, Brasil.

\section{André P. G. Machado}

Departamento de Clínica Médica. Faculdade de Ciências Médicas. Universidade do Estado do Rio de Janeiro. Rio de Janeiro, RJ, Brasil.

\section{Renata N. Aranha}

Departamento de Ginecologia e Obstetrícia. Faculdade de Ciências Médicas. Universidade do Estado do Rio de Janeiro. Rio de Janeiro, RJ, Brasil. 